\title{
Magnetic Nanomaterials in Chinese Medicine Chemical Composition Analysis and Drug Metabolism and Its Industry Prospect and Development Path Research
}

\author{
Tengfei $\mathrm{Ma}^{1}$ and Peng Liu $\mathbb{1}^{2}$ \\ ${ }^{1}$ Faculty of Management and Economics, Kunming University of Science and Technology, Kunming 650093, Yunnan, China \\ ${ }^{2}$ Faculty of Materials Science and Engineering, Kunming University of Science and Technology, Kunming 650093, Yunnan, China
}

Correspondence should be addressed to Peng Liu; better@stu.kust.edu.cn

Received 13 July 2020; Revised 24 August 2020; Accepted 19 September 2020; Published 30 September 2020

Academic Editor: Tifeng Jiao

Copyright (c) 2020 Tengfei Ma and Peng Liu. This is an open access article distributed under the Creative Commons Attribution License, which permits unrestricted use, distribution, and reproduction in any medium, provided the original work is properly cited.

\begin{abstract}
The paramagnetism and superparamagnetism of magnetic nanomaterials are very important for in vivo applications. The magnetic particles with paramagnetism or superparamagnetism can redistribute the magnetic particles after the magnetic field is withdrawn, which is widely used for the separation and purification of biomolecules. At the same time, superparamagnetic particles can also be used as MRI imaging contrast agent. Compared with Western medicine, traditional Chinese medicine is different from Western medicine in that it is originated from nature and has thousands of years of clinical efficacy. Therefore, we hope to elaborate the complex mechanism of traditional Chinese medicine through some modern technical means: through the establishment of relevant quality control system, Chinese medicine will be recognized and popularized in the international field. Therefore, this paper discusses the application of magnetic nanomaterials in the chemical composition analysis and drug metabolism of traditional Chinese medicine and its industrial prospect and development path. Firstly, the advantages of magnetic nanomaterials and the shortcomings of chemical composition analysis technology of traditional Chinese medicine are analyzed theoretically. Then, through the experimental simulation, the results show that, under the optimal conditions, the magnetic nanomaterials can be used to analyze the chemical composition of traditional Chinese medicine. The peak current and concentration of THP showed a good linear relationship in the range of $5.2 \times 10^{-8} \sim 2.1 \times 10^{-5} \mathrm{~mol} / \mathrm{L}$, and the detection limit was $1.9 \times 10^{-7} \mathrm{~mol} / \mathrm{L}$. Moreover, it showed effective results in repeatability, stability, and interference tests. Therefore, magnetic nanomaterials play an important role in the chemical composition analysis and drug metabolism of traditional Chinese medicine as well as its industrial prospect and development path.
\end{abstract}

\section{Introduction}

The magnetic core of magnetic nanomaterials is mainly composed of iron, cobalt, and other magnetic metals and their oxides. $\mathrm{Fe}_{3} \mathrm{O}_{4}$ is the first choice of magnetic nuclear materials because of its simple preparation process, easy modification, and biocompatibility. However, in the process of sample extraction, ferromagnetic oxide magnetic nanomaterials will have residual magnetism and agglomeration, which will reduce the extraction effect. Therefore, in order to prevent this phenomenon, carbon materials, silicon-based materials, and other polymer materials are often used to modify the surface of magnetic particles. This paper takes the analysis and application of nanomaterials and the development of related industries as the research premise to study the actual effects produced in the actual application process, so as to provide specific path support for the industrial transformation and upgrading of the central Yunnan urban agglomeration in Yunnan Province, China. Prior to this, the study of the specific characteristics of materials has become particularly important. After clarifying the characteristics of materials, combining regional industrial policies and actual industrial development can better serve the construction of a modern industrial system in Yunnan Province, China. This article regards the interdisciplinary research of management science and engineering disciplines and chemistry 
disciplines as an emerging discipline research direction, which is beneficial to the sustainable development of many disciplines.

Magnetic nanomaterials are different from conventional nanomaterials and magnetic materials. They refer to a kind of materials that are related to the characteristic physical length of magnetism which is just in the nanometer scale $[1,2]$. In addition to the small size effect, surface effect, and quantum size effect of nanomaterials, it also has magnetic separation, biological compatibility, superparamagnetism, and catalytic-like activity $[3,4]$. The analysis of particle morphology and structure is one of the important factors to characterize a material, powder or substance. In the scientific environment, there are several different methods to study the properties of materials, such as scanning electron microscopy, X-ray diffraction, energy-dispersive spectroscopy, and atomic force microscopy. From submicron to submicron particle, size is the final classification. Pantolimon synthesized a magnetic powder made of magnetite by coprecipitation method and analyzed it by XRD, SEM, and AFM to determine the particle/agglomeration size of the powder $[5,6]$. Teremova studied the precipitation of iron and related heavy metals in industrial wastewater and model solution using ferritization and aerobic bacterial culture. Magnetic deposits that can be extracted by magnetic separation can be produced by precipitation of iron at $p(8-10)$ and $60-80^{\circ} \mathrm{C}$ for $15-30$ minutes by magnetic separation. In the model solution, the $\mathrm{Fe}^{3+}$ precipitation with bacterial temperature of $26-34^{\circ} \mathrm{C}$ resulted in the formation of iron hydroxide nanoparticles doped with related metals. The radius of the synthesized particles is $1-5 \mathrm{~nm}$. The trihydrate iron nanoparticles are superparamagnetic in both undoped and doped groups. Teremova studied the dispersion structure, magnetic properties, and other properties of biological nanosol by atomic force microscope, small-angle X-ray scattering, X-ray diffraction, electron magnetic resonance, and Müssbauer and X-ray photoelectron spectroscopy $[7,8]$. In the study of $\mathrm{Li}$, nanosized zero-valent iron nanoparticles were coated with silica and polydopamine by two-step method. The coated nanoparticles are used as adsorbents to remove two common PAHs from aqueous system. The results show that the adsorption process is most suitable for Freundlich model and shows the characteristics of exothermic physical adsorption process. Due to their superparamagnetic properties and stability, these adsorbents can be easily collected, recycled, and reused $[9,10]$. A variety of magnetic metal nanomaterials have been successfully prepared by using $\mathrm{CaH}_{2}$ at very low temperature. Due to its strong ability of solid reducing agent, it has attracted extensive attention in the field of solid chemistry in recent years. The advantage of using $\mathrm{CaH}_{2}$ instead of $\mathrm{H}_{2}$ as reducing agent is that the reaction temperature can be reduced by several hundred degrees centigrade. The rapid decrease of reaction temperature makes the structure of nanomaterials controlled accurately, and new functionalization technologies are developed. Yamamoto briefly summarized the recent research results: $\mathrm{SiO}_{2}$-coated $\alpha$-Fe nanoparticles, biocompatible $\alpha$-Fe nanoparticles, and carbon allotropecoated $\alpha$-Fe and $\mathrm{Ni}$ nanoparticles $[4,11]$.

Drug metabolism is the main process of drug efficacy and toxicity in vivo. Develop a cheap, convenient, and rapid in vitro drug metabolism system to improve early and accurate prediction of the in vivo metabolic process, pharmacokinetics, and toxicity of candidate drugs $[13,14]$. It is important for the development and design of new drugs, administration methods and dosages, and clinical drug testing $[15,16]$. High-resolution mass spectrometry (HRMS) has become a powerful tool for the study of chemical constituents of traditional Chinese medicine. However, due to the complexity and content difference of traditional Chinese medicine compound components, it is difficult to distinguish chromatographic peaks clearly in data collection or analysis, which increases the difficulty of identification. Wang proposed a comprehensive identification strategy based on high-performance liquid chromatography linear ion trap mass spectrometry (HPLC-LMS-MS) to identify the components of traditional Chinese medicine from high-dose herbs to formula milk powder with equivalent dose. At the same time, the constituents in Xiangsha Liujunzijiajian granules were identified by mass spectrometry data, chromatographic behavior, reference standards, and previous reports. The results showed that the method can effectively and reliably identify the components of Xiangsha liujunjunjunjia decoction granules and reveal the material basis of its curative effect. This strategy provides a research idea for the characterization of multicomponent in traditional Chinese medicine prescriptions $[16,17]$. Chengqi decoction is a group of famous traditional Chinese medicine preparations. It is used to treat the symptoms caused by "internal heat," such as abdominal distension pain, difficulty in defecation, fever, and other clinical symptoms. Wen separation and identification of chemical constituents in Chinese herbal pieces were carried out by high-performance liquid chromatography electrospray ionization mass spectrometry and UPLC-TOF-MS. The results showed that 90 compounds were detected in CQS extract, including 7 anthraquinones, 39 flavonoids, 18 glycosides, 11 stilbene glycosides, 9 organic acids, 5 coumarins, and 1 lignan. Wen's research results provide a scientific basis for clinical application of CQ similar formula, and HPLC-MS can be used for systematic identification of main components in traditional Chinese medicine formula $[18,19]$. CKD affects a large proportion of the world's population, and the prevalence of CKD is increasing. At present, the standard practice is to adjust the dosage of drugs that exclude renal function when renal function declines, so as to prevent adverse drug reactions. CYPs are responsible for the metabolism of many clinical drugs. Genetics, patient factors, and drug interactions can affect CYP metabolism, resulting in changes in pharmacokinetics and drug response. There is a lot of evidence that CKD can affect the activity of many CYP isomers by circulating uremic toxins directly and/or by reducing CYP gene 
expression. There is evidence that renal transplantation can reverse the decrease of CYP metabolism in patients with end-stage renal disease and can be temporarily recovered by hemodialysis. Sugihara reviewed the effect of CKD on CYP metabolism and discussed the influence of CYP metabolic phenotype on the development of renal injury $[17,20]$. Human precise liver section is an in vitro model for acute toxicity study. However, the rapid decline of metabolic enzyme activity limits its application in the study of longterm exposure to exogenous substances. The aim of Starokozhko is to extend the activity and function of hPCLS to 5 days. Starokozhko's research shows that hPCLS cultured in Cellartis ${ }^{\circledR}$ medium is a valuable human in vitro model, which can be used for toxicological and pharmacological studies requiring long-term exogenous exposure [21, 22]. Nanobiosensor technology is also one of the many analysis methods. The unique performance of nanomaterials in various aspects is combined with the biosensor technology, which significantly improves the analytical performance of biosensors and also provides rich materials for the analysis of chemical components of traditional Chinese medicine and the research of drug metabolism [23].

Lei, a new type of CS/RGO-based composite hydrogel, was prepared by using natural polymer materials, chitosan and graphene oxide as raw materials, and glutaraldehyde as crosslinking agent. In addition, CS/GA/RGO/Pd composite hydrogels were prepared by loading palladium nanoparticles. The morphology and microstructure of the prepared hydrogel were characterized by means of SEM, TEM, XRD, TG, and BET. The catalytic performance of CS/GA/ RGO/Pd composite hydrogel was analyzed. The results showed that CS/GA/RGO/Pd composite hydrogel had good catalytic degradation performance for $p$-nitrophenol and $o$ nitroaniline. Using simple electrospinning and hydrothermal methods, new $\mathrm{MoS}_{2}$ nanosheets were prepared on $\mathrm{SrTiO}_{3}$ nanofibers and $2 \mathrm{D}$, and heterojunction NFs were synthesized by $\mathrm{SrTiO}_{3}$ and $\mathrm{MoS}_{2}$. It is necessary for Ran to study the functionalization of two-dimensional materials for the development of self-assembled nanomaterials. Ran prepared composite films based on black phosphorus and dye by Langmuir-Blodgett technique. Kaikai prepared selfassembled MXene gold nanoparticles and reported the sizeadjustable nanocomposites. Kaikai prepared nanocomposites by self-reduction of $\mathrm{MXene}_{\text {in }} \mathrm{HAuCl}_{4}$ solution at room temperature.

In this paper, by comparing with pure CNTs, the electrocatalytic activity of NCNTs on several biological small molecules was studied, and the catalytic mechanism of magnetic nanomaterials was preliminarily discussed. Based on the excellent electrocatalytic performance of NCNTs, current $\mathrm{H}_{2} \mathrm{O}_{2}$ sensors and $\mathrm{NO}$ sensors were constructed. Glucose biosensors and choline sensors were successfully constructed by detecting the $\mathrm{H}_{2} \mathrm{O}_{2}$ produced by glucose oxidase/glucose or choline oxidase/choline in the enzymatic reaction process, both of which have good detection performance. In addition, this paper initially tried a relatively mild surface functionalization method of NCNTs with the help of the special structure of pyrene molecules. This method does not destroy the original electronic structure of the material and can well maintain the biological activity of the immobilized enzyme. The construction of CYP2C9 in vitro metabolic system has potential application prospects.

\section{Magnetic Nanomaterials and Traditional Chinese Medicine}

2.1. Magnetite Nanoparticle. Magnetic iron oxide nanoparticles are particles smaller than lpm, including paramagnetic nanoparticles and superparamagnetic nanoparticles. Paramagnetism refers to the preparation of magnetic nanoparticles subject to Curie-Weiss law with the change of magnetic susceptibility with temperature and the magnetic properties of the applied substances in the biological elutriation of drug targets. On the atomic scale, the magnetic moment is heated. The effect of the disturbance is that, in the absence of an external magnetic field, these magnetic moments are randomly distributed, so that the substance does not show magnetism. When a magnetic field is applied, this magnetic moment obtains a phenomenon that there are more statistical components in the direction of the external magnetic field. Under the normal magnetic field and temperature, the energy of the magnetic moment in the external field is much smaller than the thermal disturbance energy, so the susceptibility of the paramagnetic substance is close to zero, and the value is very small, only 10-2 10-5. In a certain external magnetic field, because the thermal motion of the molecule affects the arrangement of the magnetic moments, the magnetic properties of the substance decrease with increasing temperature. The relationship between the magnetic susceptibility of paramagnetic substances and temperature is subject to Curie-Weiss law; namely,

$$
X \sigma=\frac{C}{T-\theta},
$$

Here, $C$ is called the Curie constant. $\theta$ is a constant for a certain substance, and it can be greater than, equal to, or less than zero. When $\theta=0, X \sigma=C / T$ is called Curie's law. Ferromagnetic metals are paramagnetic above the distance point; antiferromagnetic metals are paramagnetic above the Neel point. Superparamagnetism refers to a kind of magnetism that is different from the original substance itself but appears similar to paramagnetism after micronizing ferromagnetic or ferrimagnetic substance. If the ferromagnetic or ferrimagnetic material is broken into very fine single-domain particles, or the very fine single-domain particles are precipitated in the nonferromagnetic alloy matrix, when the particles are small to a certain degree, compared with the bulk ferromagnetic material, they show different properties. Suppose the magnetization of the particle is Ms and the volume is $\mathrm{n}$. The magnetic anisotropy constant is positive. Since the magnetic moment of each particle $\mu=\mathrm{MsV}$, as a whole, the magnetic moment of each particle plays the same role as the magnetic moment of each atom in a paramagnetic substance. There is a magnetic behavior of the aggregate composed of such particles and common paramagnetic substances are similar, so it is called superparamagnetic. 
The difference between super-quality and normal paramagnetism is that it is in a normal paramagnetic substance. The magnetic moment of each atom is about several Boer magnetons and in a superparamagnetic substance, the magnetic moment of each particle is about $10^{4}$ Boer magnetrons, so it can be magnetized to saturation in the usual magnetic field, superparamagnetic. An important feature is that there is no hysteresis. That is, $\mathrm{Br}$ and $\mathrm{Hc}$ are both zero.

The particle size of $\mathrm{Fe}_{3} \mathrm{O}_{4}$ magnetic particles is generally about $1-300 \mathrm{~nm}$, and different particle sizes have slightly different properties. When the particle size of the magnetic particles is less than a certain critical value, it becomes a single-domain particle assembly, and the magnetic moment changes irregularly. At this time, a superparamagnetic phenomenon occurs. $\mathrm{Fe}_{3} \mathrm{O}_{4}$ magnetic particles have been reported to be biocompatible, and based on their unique physical and magnetic properties, $\mathrm{Fe}_{3} \mathrm{O}_{4}$ magnetic particles have been widely used in the medical field. Therefore, $\mathrm{Fe}_{3} \mathrm{O}_{4}$ magnetic particles are expected to become one of the most promising materials in the medical field.

2.2. Chinese Medicine Chemical Composition. The components of traditional Chinese Medicine analyzed by the current-type electrochemical sensor need to have active groups which can produce redox. According to the structural formula analysis of the components, it can be found that most of them have active groups and have the potential to be determined by electrochemistry. The quality of traditional Chinese medicine is affected by many uncontrollable factors: in the process of cultivation and storage, in order to prevent and control diseases and insect pests, pesticides will be sprayed on Chinese medicinal materials, and the growth process will also be affected by the soil quality of the growing environment; illegal traders will add contraband in order to increase profits and sulfur fumigation in order to make the color of traditional Chinese medicine bright and lustrous.

The separation and purification of traditional Chinese medicine has been developed for a long time. There are many separation methods commonly used in industry, and different methods are selected for different compounds. Highspeed countercurrent chromatography (HSCCC) is a new type of liquid-liquid partition chromatography technology. It is characterized by the separation of separated substances in two liquid phases, which overcomes the sample defects brought by traditional solid-phase carriers, and provides a new way for the separation and preparation of traditional Chinese medicine components and the analysis and identification of traditional Chinese medicine. The basic principle of high-performance liquid chromatography (HPLC) is based on the different partition coefficients of different substances in the chromatographic column, so as to achieve the separation effect. This method has many advantages, which is suitable for the separation and purification of multicomponent complex mixture. $80 \%$ of the compounds can be used. However, the chromatograph is expensive, and the column is consumable, easy to pollute, and high in application cost. Macroporous resin has macroporous network structure, which has the characteristics of large specific surface area and high adsorption efficiency. There is no exchange group on the surface, which belongs to physical adsorption. There are two main separation mechanisms: adsorption and molecular sieve. The main advantages of macroporous resin are low application cost, and the resin can be used repeatedly; large sample size, suitable for the treatment of large quantities of samples, and has a high enrichment effect; macroporous resin can also remove nonpolar and polar impurities such as chlorophyll and sugar in traditional Chinese medicine; according to the polarity of solvent elution, the target compounds can also be separated by polarity.

Alkaloids are a kind of basic organic compounds containing nitrogen, which are similar to alkali. Most of them have complex ring structure. Nitrogen is contained in the ring and has significant biological activity. Flavonoid compounds generally refer to a series of compounds formed by connecting two benzene rings (A- and B-ring) with phenolic hydroxyl group through the central three carbon atoms, and the basic mother nucleus is 2-phenylchromone. Flavonoids are often linked with active functional groups. It is possible for the electrochemical determination of flavonoids; terpenoids refer to hydrocarbons and their oxygencontaining derivatives whose molecular formula is multiple of isoprene units in nature, and the unsaturated bond in terpenoids structural formula provides the possibility for electrochemical detection; sugars are an important class of organic compounds widely distributed in nature. It plays an important role in the process of life activities and is the main source of energy for all living bodies to maintain life activities. Glycosides, also known as glycosides, are compounds formed by the end group carbon atoms of sugars or sugar derivatives connected with another class of non-sugar substances.

\subsection{Application of Magnetic Nanomaterials Combined with} Electrochemical Sensors in the Analysis of Traditional Chinese Medicine. A large number of synthetic drugs play an undeniable role in the field of medicine, but their use is often accompanied by side effects that are difficult to predict. From the structure analysis, most of them have electrochemical active groups. In principle, all the active components of traditional Chinese medicine containing these electrochemical active groups can be studied by a suitable electrochemical technology, so as to realize quantitative analysis and infer the relevant mechanism. At present, carbon materials are the most widely studied and used materials. From the macro perspective, there are three kinds of allotropic carbon, namely, graphite, diamond, and amorphous carbon. Graphene is a single-layer $\mathrm{sp}^{2}$ hybrid two-dimensional crystal with hexagonal honeycomb. Graphene is composed of single-layer carbon atoms, which is a new type of carbon nanomaterials. The thickness of single-layer graphene is only $0.34 \mathrm{~nm}$, and the theoretical surface area can reach $2600 \mathrm{~m}^{2} / \mathrm{g}$, and it has 
outstanding thermal conductivity and mechanical properties. In addition, the $\mathrm{sp}^{2}$ structure of graphene makes it have high electron mobility and a series of excellent physical and chemical properties, such as good quantum tunneling effect. Graphene oxide has a SP2 plane structure without reduction and contains a large number of oxygencontaining groups. These active groups were used to open the ring of graphene, and the modified group was grafted onto the surface of graphene in the form of covalent bond, so as to realize the covalent functional modification of graphene. Covalent functionalization can not only improve the dispersion of graphene in solvent, but also prepare a series of novel graphene hybrid materials for various fields, which broaden the application range of graphene. In addition to covalent functionalization of graphene, $\pi-\pi$ bond, ionic bond, and hydrogen bond can also be used for noncovalent functionalization of graphene. The noncovalent functionalized modification will not destroy the n-electronic structure of graphene, and the original characteristics of graphene can be maintained by using the principle of physical adsorption or polymer coating on graphene surface. Carbon nanotubes (CNTs) are hollow nanotubes composed of single- or multilayer graphite-like planes composed of six membered rings. Each carbon atom of carbon nanotubes is bonded to three carbon atoms by $\mathrm{sp}^{2}$ hybridization. Because carbon nanotubes are round tubes made of single- or multilayer flake graphite, carbon nanotubes inherit the excellent physical and chemical properties of graphite and have many unique properties.

\section{Experiment Materials and Methods}

3.1. Experimental Materials. Eliminate residues 1-290 from the NOS oxygenase region to obtain D290nNOSoxy, with a molecular weight of about $49 \mathrm{kDa}$. Phosphate buffer solution is prepared by mixing $\mathrm{Na}_{2} \mathrm{HPO}_{4} \cdot 12 \mathrm{H}_{2} \mathrm{O}$ and $\mathrm{NaH}_{2} \mathrm{PO}_{4} \cdot 2 \mathrm{H}_{2} \mathrm{O}$ solutions. Other reagents are analytically pure, and all solutions are prepared with double distilled water.

\subsection{Connection of Magnetic Nanoparticles and Traditional} Chinese Medicine. Add $150 \mathrm{mg}$ of the prepared polymercontaining magnetic particles to $2 \mathrm{ml}$ of ethylenediamine buffer to mix, then add $40 \mathrm{mg}$ of carbodiimide, react at room temperature for 3 hours, dialyze 4 times with $1000 \mathrm{ml}$ of $10000 \mathrm{MCW} 0$ dialysis bag to remove excess ethylenediamine and salt, and freeze-dry to obtain magnetic particles crosslinked with high-molecular-weight polymer and active group NH2. Use EDC as the linking agent to prepare $20 \mathrm{mg}$ of surface-crosslinked magnetic particles, in $1 \mathrm{ml}$ of the connection buffer, prepare $2 \mathrm{mg}$ of salvianolic acid B in the connection buffer, prepare $10 \mathrm{mg} / \mathrm{ml}$ EDC connection buffer, react at room temperature for 3 hours, and dialyze with 10000MCW0. The bag was dialyzed 5 times with $1000 \mathrm{ml}$ of $0.85 \% \mathrm{NaCl}$ aqueous dialysis solution and lyophilized. Take a small amount of lyophilized sample and salvianolic acid B for infrared detection.

\section{Application of Magnetic Nanomaterials in Chemical Composition Analysis and Drug Metabolism of Traditional Chinese Medicine}

4.1. Determination and Analysis of THP by DPV. The comparison results of the electrochemical method and other THP determination methods are shown in Table 1.

Compared with other methods, the electrochemical method was chosen because of its wider linear range, lower LOD, and easier sample processing. As the two electrochemical measurement methods of THP, SWV only preliminary studies the electrochemical characteristics of THP. The method used in this paper has made an in-depth discussion on the optimal conditions of THP and deduced its electrochemical mechanism. In the measurement of actual samples, the method adopted in this paper has shown satisfactory results. The differential pulse voltammograms of NiNPs/SGS/GCE at different concentrations of $0.5-20.0 \mu \mathrm{M}$ THP are shown in Figure 1.

Under optimized conditions, the DPV of NiNPs/SGS/GCE at different concentrations of $5.0 \times 10^{-7}-2.0 \times 10^{-5} \mathrm{~mol} / \mathrm{L}$ THP, Ipa has a linear relationship with the concentration: Ipa $(\mu \mathrm{A})=0.263 c+0.555(\mu \mathrm{M}, r=0.9988)$. The LOD of THP of this modified electrode was $1.9 \times 10-7 \mathrm{~mol} / \mathrm{L}$. By comparing bare GCE, SGS/GCE, and NiNPs/GCE, NiNPs/SGS/GCE further increased the oxidation peak current of THP and promoted the electrochemical reaction of THP on the modified electrode. The optimal conditions of THP were optimized by using CV and DPV, such as $\mathrm{pH}$, modification amount, resting time of electrochemical deposition, and scanning rate. Under the best conditions, the peak current of the modified electrode to THP and the concentration showed a good linear relationship in the range of $5.2 \times 10^{-8} \sim 2.1 \times 10^{-5} \mathrm{~mol} / \mathrm{L}$, and the detection limit reached $1.9 \times 10^{-7} \mathrm{~mol} / \mathrm{L}$, and in repeatability, stability, and interference tests, the results are satisfactory.

4.2. Sample Recovery Analysis. Prepare three groups of high-, medium-, and low-concentration drug model sample solutions, each group was tested in parallel for 5 times to calculate and analyze it. The results of the determination of the recovery rate of drug solutions with different concentrations are shown in Table 2.

According to the results in the table, the sample recovery rate of the drug models of each concentration is between $98.7 \%$ and $101.7 \%$, and the relative standard deviation is between $2.18 \%$ and $3.36 \%$. The UV-vis spectra of MP-1, MP2 , and MP-3 after adsorption of the drug model rutin and anacardin are shown in Figure 2.

The analysis of the UV test results shows that the MP-1, MP-2, and MP-3 prepared have a certain amount of adsorption effect on the drug model, but the adsorption amount of each magnetic particle to the drug model is different, which is mainly caused by the magnetic property. The adsorption principle between particles and drug model molecules is different. MP-1 has a certain adsorption effect on drug model molecules. The main adsorption is physical adsorption. Second, there is a little $\mathrm{Fe}^{3+}$ on the surface of MP-1. $\mathrm{Fe}^{3+}$ can form a complex with catechol structure compounds through metal 
TABLE 1: Comparison of electrochemical method with other THP determination methods.

\begin{tabular}{lccc}
\hline Test method & The measuring object & Concentration range $(\mu \mathrm{M})$ & LOD $(\mu \mathrm{M})$ \\
\hline LC-MS/MS & L-THP & $2.84 \times 10^{-4}-2.84$ & $2.84 \times 10^{-4}$ \\
LC-ESI-MS & THP & $2.84 \times 10^{-3}-1.43$ & $2.81 \times 10^{-3}$ \\
UPLC-FLD & L-THP and cocaine & $7.05 \times 10^{-3}-0.72$ & $7.05 \times 10^{-3}$ \\
LC-QTQF/MS & $(+)$-THP and (-)-THP & $1.45 \times 10^{-2}-70.5$ & $1.45 \times 10^{-2}$ \\
(CNTS/GCE) by SWV & THP & $0.9-50.3$ & 0.4 \\
(NiNPs/SGS/GCE) by DPV & THP & $0.51-20.3$ & 0.19 \\
\hline
\end{tabular}

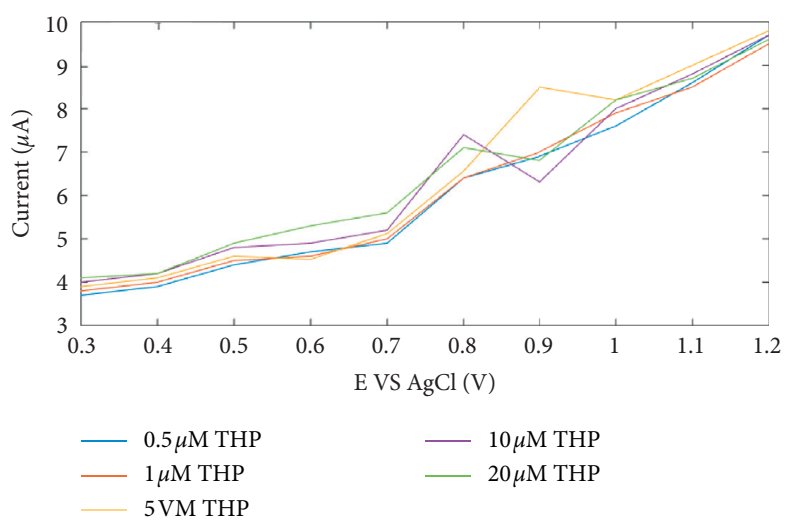

Figure 1: Differential pulse voltammetry of NiNPs/SGS/GCE at different concentrations of $0.5-20.0 \mu \mathrm{m}$ THP.

TABLE 2: Determination results of recovery rate of drug solution with different concentrations.

\begin{tabular}{lcccc}
\hline Sample & $\begin{array}{r}\text { Added } \\
(\mathrm{mg} / \mathrm{L})\end{array}$ & Found (mg/L) & Recovery (\%) & RSD (\%) \\
\hline Rutin & 6.37 & 6.58 & 101.8 & 2.17 \\
Rutin & 15.16 & 14.92 & 98.8 & 3.17 \\
Rutin & 19.51 & 19.34 & 99.13 & 2.05 \\
9.76 & 9.78 & 9.98 & 102.3 & 2.49 \\
Rhoifolin & 17.46 & 17.69 & 101.7 & 2.34 \\
Rhoifolin & 30.45 & 29.98 & 98.7 & 3.41 \\
\hline
\end{tabular}

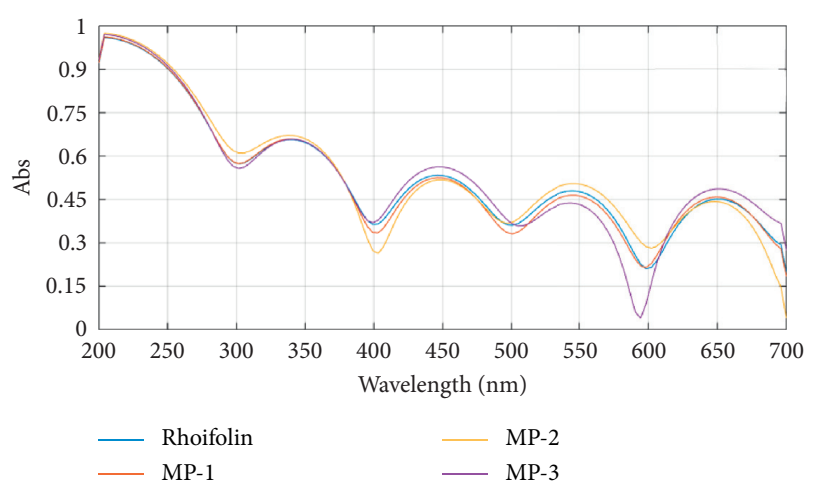

Figure 2: The UV-vis spectra of MP-1, MP-2, and MP-3 after adsorption of the drug model rutin and anacardin.

coordination. Compound MP-2, which has a certain adsorption effect on drug model molecules, adsorbs more on drug model molecules than MP-1, because it has rich $\mathrm{Fe}^{3+}$ on the surface of it, which can be matched with drug model molecules through metals. The site forms a complex and is adsorbed. The adsorption capacity of MP-3 to drug model molecules is much greater than that of MP-1 and MP-2 to drug model molecules. In addition to appealing the two adsorption effects, the most important adsorption is the effect caused by the boric acid modified on the surface of MP-3. Using the specific recognition effect of boric acid, the cyclic lactone structure is formed by the reversible covalent bond with the drug model molecule, so that the drug model is adsorbed and separated from the solution.

4.3. Analysis of Magnetic Nanomaterials in Chinese Medicine Chemical Composition Analysis and Drug Metabolism and Its Industry Prospects and Development Path. The synthesis process of magnetic nanocomposites can be characterized by UV-vis absorption spectrum. The UV-vis absorption spectrum of centrifugal supernatant and $\mathrm{PBA} / \mathrm{G}$ is shown in Figure 3.

After continuous washing/centrifugation cycles, the absorption peak of free PBA in the supernatant gradually weakened. After three washings, the absorption peak almost disappeared completely, indicating that free PBA molecules have been completely removed from $\mathrm{PBA} / \mathrm{G}$ and $\mathrm{PBA} /$ NCNTs. At this time, the UV-vis spectra of PBA/G and PBA/ NCNTs solutions still have obvious PBA absorption peaks. This shows that this method can successfully assemble PBA molecules on the surface of graphene and NCNTs. The carbon nanomaterials dispersed in the solution will reduce the light transmittance of the solution and increase the background. The modification of PBA molecules on NCNTs also reduced the redox peak on NCNTs/GC electrode. It can be seen that magnetic nanomaterials have practical value in the analysis of traditional Chinese medicine chemical 


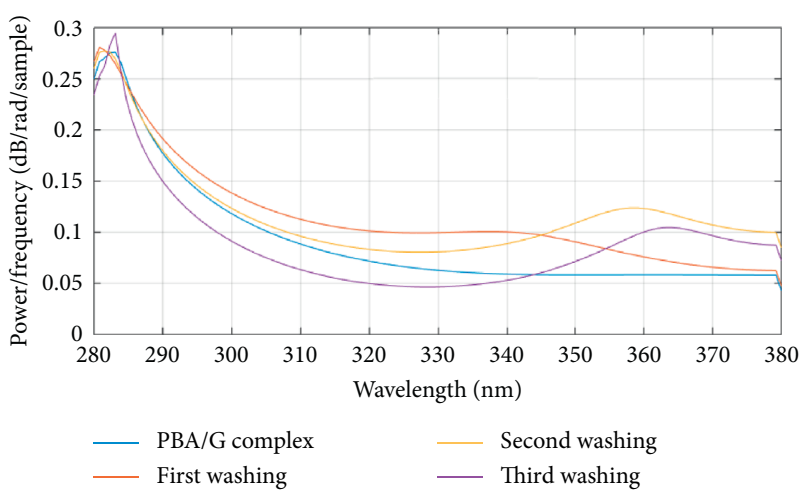

FIGURE 3: UV-vis absorption spectra of centrifuged supernatant and $\mathrm{PBA} / \mathrm{g}$.

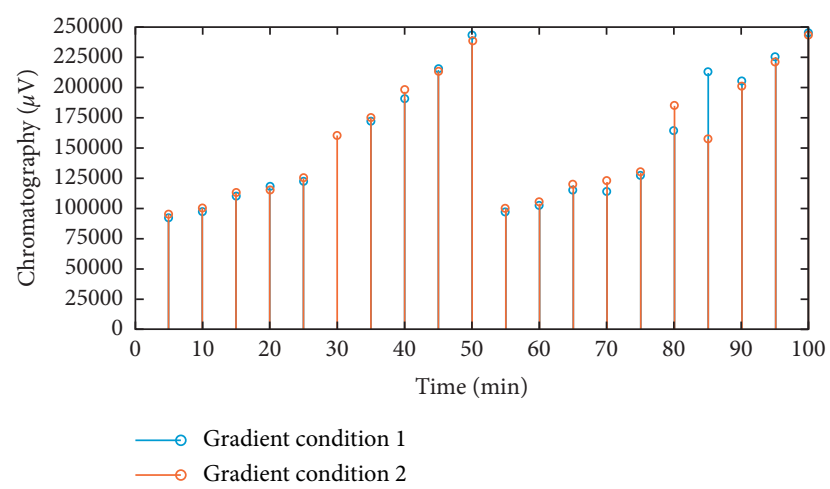

Figure 4: Comparison of chromatograms under two gradient conditions.

composition and drug metabolism, and magnetic nanomaterials have important research value for the industrial prospect and development path of traditional Chinese medicine chemical composition analysis.

\subsection{Optimization Analysis of Separation and Analysis Con-} ditions of Scutellaria baicalensis. The comparison results of the two gradient conditions chromatograms are shown in Figure 4.

It can be seen that the chromatogram analysis time of the gradient condition 1 is short, saving the use of mobile phase, and the separation effect is equivalent to the gradient condition 2, so the gradient condition 1 is selected as the Scutellaria chromatogram analysis condition. The molecular structure of baicalin contains orthophenolic hydroxyl- and carboxyl-like structures, baicalein molecular structure contains orthophenolic hydroxyl groups, and baicalin contains orthophenolic hydroxyl- and carboxyl-like structures, which can specifically adsorb to magnetic nanoparticles. However, baicalein only has metaphenolic hydroxyl groups and cannot specifically adsorb to magnetic nanoparticles. The volume ratio of Scutellaria baicalensis extract and magnetic nanoparticles is set to 5:1. The main components of Scutellaria baicalensis can be absorbed completely, saving the use of raw materials to the greatest extent, and the components in the desorption supernatant contain hydroxyl and carboxyl groups. The specific adsorption capacity of phenolic hydroxyl and carboxyl groups was verified.

\section{Conclusions}

In this paper, the magnetic nanoparticles are used as the adsorption carrier, and the Chinese herbal medicine is the research object. The selective adsorption of alkylphenols, flavonoids, and cyclotriterpenoids was investigated. Through the analysis of the standard map and characterization results, we obtained the fact that magnetic nanoparticles have specific adsorption for orthophenolic hydroxyl and carboxyl groups and can desorb the target substance under the action of eluent to achieve the purpose of separation and purification. In this paper, new nanorodlike polymers are used as templates to synthesize magnetic nanoparticles. The polymer has abundant carboxyl groups, which provides a template for the synthesis of magnetic particles. After the synthesis is completed, the surface of the magnetic particles contains excess free carboxyl groups and the branched structure of the polymer, which can prevent the aggregation of particles. Suspension stability plays an important role in the suspension. This article combines the screening of the target of active ingredients of traditional Chinese medicine and the technology of phage display library to study the mechanism of active ingredients of traditional Chinese medicine on the molecular level and attempts to further study the mechanism of active ingredients of traditional Chinese medicine. In this paper, the preparation technology of nano new magnetic materials is organically integrated into medical technology, which provides a good research basis for the combination of magnetic nano medical technology. Aiming at the application of this material in chemiluminescence bioanalysis, combined with the current status of the industry in the central Yunnan city group, it is of great significance to the development prospects of the material industry. On the basis of the early industrial economic development research, combined with the practice of constructing a modern industrial system in Yunnan Province, China, it is found that the nanomaterial industry is a good breakthrough in the process of industrial transformation and upgrading of the central Yunnan urban agglomeration. It also provides a research basis for the analysis of magnetic nanomaterials in the analysis of chemical composition of traditional Chinese medicine and drug metabolism and its industrial prospect and development path. This article discusses the possibility of the integration of materials' chemistry and industrial development from the perspective of chemistry. In the author's research on the industrial development of the central Yunnan urban agglomeration in Yunnan Province, China, we will further study the interdisciplinary research of management science and engineering and chemistry.

\section{Data Availability}

The data used to support the findings of this study are included within the article. 


\section{Conflicts of Interest}

The authors declare that there are no conflicts of interest regarding the publication of this paper.

\section{References}

[1] X. Chen and L. Zhang, "A review on micromixers actuated with magnetic nanomaterials," Microchimica Acta, vol. 184, no. 1, 11 pages, 2017.

[2] A. H. Qi, A. Z. Li, A. H. Zheng, and Q. Jia, "Facile preparation of hydrophilic glutathione modified magnetic nanomaterials for specific enrichment of glycopeptides," Chinese Chemical Letters, vol. 30, no. 12, pp. 2181-2185, 2019.

[3] D. Zhao, J. Li, and M. Wang, "Iron-based magnetic nanomaterials and their environmental applications," Critical Reviews in Environmental Science and Technology, vol. 46, no. 8, pp. 783-826, 2016.

[4] H. Yamamoto, L. Seinberg, and H. Kageyama, "Low temperature synthesis of magnetic metal nanomaterials by using calcium hydride," Journal of the Japan Society of Powder and Powder Metallurgy, vol. 64, no. 4, pp. 147-154, 2017.

[5] M. C. Pantilimon, C. I. Dragan, C. Gradinaru et al., "Morphological analysis of magnetic nanomaterials through comparative methods," UPB Scientific Bulletin, Series B: Chemistry and Materials Science, vol. 80, no. 3, pp. 175-182, 2018.

[6] C. Song, L. ü Yi, and R. Wu, "Application prospect of nanomaterials mediated magnetic technology in the diagnosis and treatment of surgical diseases," Chinese Science Bulletin, vol. 65 , no. 13, pp. 1251-1263, 2020.

[7] M. I. Teremova, E. A. Petrakovskaya, A. S. Romanchenko et al., "Ferritization of industrial waste water and microbial synthesis of iron-based magnetic nanomaterials from sediments," Environmental Progress \& Sustainable Energy, vol. 35, no. 5, pp. 1407-1414, 2016.

[8] H. Fu, J. Hu, H. Y. Zhang et al., "Preparation of the magnetic mesoporous silica nanomaterials with formic acid solution as the medium and its adsorption properties," Journal of East China University of Ence \& Technology, vol. 43, no. 2, pp. 207-212, 2017.

[9] M. Lei, Q. Zhou, and Y. Liu, "Recyclable nanoscale zero-valent iron-based magnetic polydopamine coated nanomaterials for the adsorption and removal of phenanthrene and anthracene," Science and Technology of Advanced Materials, vol. 18, no. 1, pp. 3-16, 2017.

[10] C. R. Laili and S. Hamdan, "Heating behaviour of iron oxide nanomaterials via magnetic nanoparticle hyperthermia," Asian Journal of Chemistry, vol. 28, no. 12, pp. 2675-2679, 2016.

[11] D. J. Yue, Y. Jin, and D. J. Sellmyer, "Effect of boron addition on magnetic-domain structure of rapidly quenched Zr2Co11-Based nanomaterials," MRS Advances, vol. 1, no. 34, pp. 2379-2385, 2016.

[12] R. Olsson, P. Aadal Nielsen, and F. Ek, “An ex vivo model for evaluating blood-brain barrier permeability, efflux, and drug metabolism," ACS Chemical Neuroscience, vol. 7, no. 5, pp. 668-680, 2016.

[13] K. K. Filipski, M. A. Pacanowski, and A. N. Freedman, "Dosing recommendations for pharmacogenetic interactions related to drug metabolism," Pharmacogenetics and Genomics, vol. 26, no. 7, pp. 334-339, 2016.

[14] M. A. López-García, I. A. Feria-Romero, H. Serrano et al., "Influence of genetic variants of CYP2D6, CYP2C9, CYP2C19 and CYP3A4 on antiepileptic drug metabolism in pediatric patients with refractory epilepsy," Pharmacological Reports, vol. 69, no. 3, pp. 504-511, 2017.

[15] A. A. Oyagbemi, D. Bester, J. Esterhuyse et al., "Evaluating the role of drug metabolism and reactive intermediates in trazodone-induced cytotoxicity toward freshly-isolated rat hepatocytes," Drug Research, vol. 66, no. 11, pp. 592-596, 2016.

[16] X. Tang, Q. Zhang, and Z. Lu, "Identification of chemical constituents in traditional Chinese medicine formula using HPLC coupled with linear ion trap-orbitrap MS from high doses of medicinal materials to equivalent doses of formula: study on Xiang-Sha-Liu-Jun-Zi-Jia-Jian granules," Journal of Separation Science, vol. 39, no. 9, pp. 1619-1627, 2016.

[17] Y. Sugihara, K. I. Watanabe, and A. Végvári, "Novel insights in drug metabolism by MS imaging," Bioanalysis, vol. 8, no. 6 , pp. 575-588, 2016.

[18] X. Fan, K. Luo, and S. Xiao, "Qualitative analysis of chemical constituents in traditional chinese medicine analogous formula cheng-Qi decoctions by liquid chromatography-mass spectrometry," Biomedical Chromatography, vol. 30, no. 3, pp. 301-311, 2016.

[19] C. Swart and C. Dandara, "MicroRNA mediated changes in drug metabolism and target gene expression by efavirenz and rifampicin in vitro: clinical implications," OMICS: A Journal of Integrative Biology, vol. 23, no. 10, pp. 496-507, 2019.

[20] M. A. Ladda and K. B. Goralski, "The effects of CKD on cytochrome P450-mediated drug metabolism," Advances in Chronic Kidney Disease, vol. 23, no. 2, pp. 67-75, 2016.

[21] D.-C. Hao and P.-g. Xiao, "Impact of drug metabolism/ pharmacokinetics and their relevance upon traditional medicine-based cardiovascular drug research," Current Drug Metabolism, vol. 20, no. 7, pp. 556-574, 2019.

[22] G. M. M. Groothuis, S. Vatakuti, and B. H. Schievink, "Maintenance of drug metabolism and transport functions in human precision-cut liver slices during prolonged incubation for 5 days," Archives of Toxicology, vol. 91, no. 5, pp. 2079-2092, 2016.

[23] K. Thiengsusuk, K. Boonprasert, and K. Na-Bangchang, "A systematic review of drug metabolism studies of plants with anticancer properties: approaches applied and limitations," European Journal of Drug Metabolism and Pharmacokinetics, vol. 45, no. 2, pp. 173-225, 2020. 